\title{
Obtenção de suco de caju atomizado através do controle das condições de secagem
}

\author{
Érica M. F. F. Rocha ${ }^{1}$, Sanyelle L. Sousa ${ }^{2}$, Janaina de P. da Costa ${ }^{3}$, \\ Sueli Rodrigues ${ }^{4}$, Marcos R. A. Afonso ${ }^{5} \&$ José M. C. da Costa ${ }^{6}$ \\ ${ }^{1}$ IFRN. Pau dos Ferros, RN. E-mail: emffrocha@yahoo.com.br (Autor correspondente) \\ ${ }^{2}$ UFC. Fortaleza, CE. E-mail: sanyellelima@yahoo.com.br \\ ${ }^{3}$ UFC. Fortaleza, CE. E-mail: eng.janaina@yahoo.com.br \\ ${ }^{4}$ UFC. Fortaleza, CE. E-mail: sueli@ufc.br \\ ${ }^{5}$ UFC. Fortaleza, CE. E-mail: m.r.a.afonso@gmail.com \\ ${ }^{6}$ UFC. Fortaleza, CE. E-mail: correiacostaufc@gmail.com
}

\section{Palavras-chave:}

spray-dryer

condições operacionais

qualidade

\begin{abstract}
R E S U M O
O suco de caju é um produto amplamente consumido no Brasil, sobretudo na região Nordeste. Sua desidratação pelo processo de spray-dryer se mostra como alternativa relevante para aproveitar o excedente de produção. Dentro deste contexto este trabalho tem, como objetivo, estabelecer a melhor condição operacional de secagem em spray-dryer visando à obtenção de suco de caju em pó através de um planejamento composto central rotacional. Também foi realizada a caracterização físico-química do suco integral quanto do suco em pó de caju obtido através da melhor condição operacional do planejamento. O planejamento experimental utilizado não apresentou diferença significativa ao nível de 0,05 de probabilidade revelando que o modelo não foi ajustado aos dados, porém é possível se observar, qualitativamente, uma zona ótima de processo. Os principais resultados dos parâmetros físicos e físico-químicos para o suco integral de caju e o suco em pó no que se refere à umidade, $\mathrm{pH}$, acidez titulável, sólidos solúveis, vitamina $\mathrm{C}$

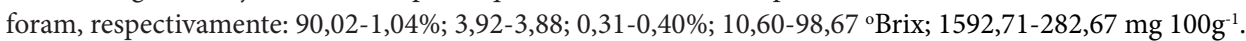

Key words: spray-dryer operational conditions quality

\section{Obtention of cashew juice atomized by controlling the drying conditions}

\begin{abstract}
A B S T R A C T
Cashew juice is a product that is widely consumed in Brazil, mainly in northeast Brazil. Dehydrating this juice by spray-drying is an important alternative to use the production excess. Given the above, this study aimed to establishing the best spray-drying operational conditions to obtain the powder cashew, by means of a rotational center composite design. The physicochemical characterization of both the integral juice and the powder juice, obtained from the best operational conditions was also carried out. The experimental design used did not show any significant difference at 0.05 level of probability, which highlighted that the model was not adjusted to the data. However, it was possible to observe a qualitative optimum in the process. The main results for physical and physicochemical parameters for both the integral cashew juice and the powder juice, concerning humidity, $\mathrm{pH}$, titratable acidity, soluble solids, vitamin $\mathrm{C}$ were, respectively: $90.02-1.04 \% ; 3.92-3.88 \% ; 0.31-0.40 \% ; 10.60-98.67$ 'Brix; 1592.71-282.67 mg 100g-1.
\end{abstract}

\section{INTRODUÇÃo}

Conforme Andrigueto et al. (2006), o Brasil é o terceiro maior produtor de frutas com $6 \%$ da produção mundial dentre as quais se encontra o caju, que é uma fruta tropical cuja produção interna está concentrada no Nordeste sendo de grande importância social e econômica para esta região (Cianci et al., 2005). Confirmando esses dados e de acordo com o IBGE (2012) foram produzidos, no ano de 2012, 80.630 toneladas de castanha de caju no Brasil sendo a Região Nordeste responsável por 95,28\% desta produção. Segundo Estrella (2004) o consumo de suco de frutas foi de aproximadamente 2,2 bilhões de litros, nas mais diferentes formas destacando-se, dentre eles, $579 \mathrm{mil}$ $\mathrm{L}$ de sucos integrais com destaque para o caju (51\%).

A secagem em spray-dryer é um processo amplamente utilizado na indústria de alimentos, que pode ser utilizada para transformar a polpa/ suco de fruta na forma de pó, permitindo um armazenamento prolongado e uma maior estabilidade e longevidade do produto, de modo a ter uma maior vida útil, podendo o consumidor ter acesso a esse produto durante o ano, e não apenas no período de safra da fruta (Anselmo et al, 2006). Este processo tem-se mostrado, quando aplicado nas condições ideais, eficaz na obtenção de diversos produtos (Adhikari et al., 2004). No entanto, é imprescindível que se faça 
sua otimização a fim de se obter produtos na forma de pó com melhores características sensoriais e nutricionais tal como se obter bom rendimento do referido processo.

Com base no exposto objetivou-se, neste trabalho, estabelecer a melhor condição operacional de secagem em spray-dryer para obtenção do pó do suco integral de caju e a caracterização física e físico-química do suco integral de caju e seu pó.

\section{Material e Métodos}

O suco integral de caju sem adição de conservantes, foi processado na planta-piloto de processamento de frutas da Embrapa Agroindústria Tropical localizada na cidade de Fortaleza, CE. Após o processo o suco foi embalado em embalagem de poliestireno tereftalato (PET) e conduzido ao Laboratório de Controle de Qualidade de Alimentos e Secagem do Departamento de Tecnologia de Alimentos da Universidade Federal do Ceará. O suco foi mantido sob congelamento em freezer vertical a $-18^{\circ} \mathrm{C}$ e o descongelamento foi realizado na embalagem original do produto, em geladeira $\left(5^{\circ} \mathrm{C}\right)$ por $18 \mathrm{~h}$.

Após o descongelamento foram pesados $600 \mathrm{~g}$ do suco integral para cada ensaio e adicionados 30\% (p/p) de maltodextrina. Após completa dissolução e homogeneização a solução formulada foi levada ao spray-dryer modelo LM MSD 1.0 da marca Labmaq do Brasil com a utilização de um bico aspersor de 1,2 $\mathrm{mm}$ de abertura de diâmetro, para início do processo de atomização obedecendo a parâmetros também estabelecidos no planejamento experimental tendo, como variáveis independentes, a vazão de ar quente $\left(\mathrm{m}^{3} \mathrm{~min}^{-1}\right)$ e a temperatura de entrada do ar $\left({ }^{\circ} \mathrm{C}\right)$ e como variáveis respostas (rendimento de processo com resultado expresso em porcentagem e teor de umidade do pó obtido). Foram mantidos constantes os parâmetros: velocidade de alimentação $(0,55$ $\mathrm{L} \mathrm{h}^{-1}$ ) e vazão do ar comprimido (3,0 $\left.\mathrm{L} \mathrm{min}^{-1}\right)$.

Após a secagem os pós foram pesados para efeito de rendimento e acondicionados em sacos de filme plástico, de dimensões 22 x $50 \mathrm{~cm}$, formados da combinação de poliamida (PA) e polietileno (PE). Os pós foram acondicionados a vácuo em seladora da marca Fun Kitchen, modelo BR 2205, e mantidos sob temperatura ambiente sem a presença de luz.

Para os pós do suco integral de caju foi realizada a análise de rendimento (variável resposta utilizada na análise de significância do planejamento), calculada através da Eq. 1.

$$
\mathrm{R}(\%)=\frac{\mathrm{A}}{\mathrm{B}} \times 100
$$

sendo:

A - quantidade de produto (pó) obtido após o processo menos o resultado da análise sua umidade, $g$

B - quantidade total, de sólidos presentes no suco de caju mais a maltodextrina adicionada, $g$

Todas as determinações físico-químicas, tanto no suco integral quanto no suco em pó, foram realizadas em triplicata utilizando-se a melhor condição de processo obtida no planejamento. As análises foram as seguintes: umidade (determinada utilizando-se uma estufa a vácuo a $70{ }^{\circ} \mathrm{C}$, sob pressão reduzida, até massa constante), $\mathrm{pH}$ (determinado através de um medidor de $\mathrm{pH}$, calibrado periodicamente com soluções tampão de pH 4,0 e 7,0), acidez titulável (determinada por titulação com $\mathrm{NaOH} 0,1 \mathrm{~N}$ e os resultados expressos em percentagem de ácido cítrico) e os sólidos solúveis (determinados através da leitura dos ${ }^{\circ}$ Brix a $20{ }^{\circ} \mathrm{C}$ em refratômetro com escala variando de 0 a $90{ }^{\circ} \mathrm{Brix}$ ) foram realizadas segundo a metodologia do IAL (2004).

$\mathrm{O}$ conteúdo de vitamina $\mathrm{C}$ foi determinado através do método titulométrico baseado na redução do indicador 2,6-diclorofenolindofenol pelo ácido ascórbico em que os resultados foram expressos em mg de ácido ascórbico por $100 \mathrm{~g}$ de amostra (AOAC, 1997).

A análise de cor foi realizada através do colorímetro Minolta modelo Chroma-Meter CR-410 com determinação da escala CIE $L^{*} a^{*} b^{*}$, que inclui as variáveis $L^{*}, a^{*}, b^{*}$, Chroma $\left(C^{*}\right)$, ângulo Hue $\left(\mathrm{H}_{0}^{*}\right)$ sendo as coordenadas $\mathrm{a}^{\star} \mathrm{e} \mathrm{b}^{\star}$ utilizadas para calcular a diferença de cor $\left(\Delta \mathrm{EL}^{\star} \mathrm{a}^{\star} \mathrm{b}^{\star}\right)$ através da Eq. 2 :

$$
\Delta \mathrm{EL}^{*} \mathrm{a}^{*} \mathrm{~b}^{*}=\sqrt{\left[\left(\mathrm{L}^{*}-\mathrm{L}_{0}^{*}\right)^{2}+\left(\mathrm{a}^{*}-\mathrm{a}_{0}{ }^{*}\right)^{2}+\left(\mathrm{b}^{*}-\mathrm{b}_{0}{ }^{*}\right)^{2}\right]}
$$

em que:

$\mathrm{L}^{*}$ e $\mathrm{L}_{0}^{*}$ - luminosidade das amostras secas e frescas, respectivamente

$\mathrm{a}^{*}$ e $\mathrm{a}_{0}^{*}$ - Intensidade de cor vermelha das amostras secas e frescas, respectivamente

$\mathrm{b}^{*} \mathrm{e} \mathrm{b}_{0}^{*}$ - Intensidade de cor amarela das amostras secas e frescas, respectivamente

A análise de significância dos efeitos das variáveis independentes sobre o parâmetro de rendimento (variável resposta) foi avaliada através do programa Statistica, versão 7,0 (StatSoft Co., 2007) tal como, também, a análise de variância (ANOVA) dos resultados dos parâmetros físico-químicos avaliados. Neste contexto o teste F foi empregado como critério de validação da significância estatística dos modelos obtidos a nível de confiança de $95 \%$.

\section{Resultados e Discussão}

Na Tabela 1 se encontram o planejamento composto central rotacional e os resultados médios do parâmetro de rendimento de processo e teor de umidade do pó obtido podendo-se observar que os valores de rendimento variaram de 73,98\% (Ensaio G) a 99,30\% (Ensaio H) nesses dois ensaios utilizou-se o mesmo valor da vazão de ar quente demonstrando grande efeito da temperatura sobre esta variável resposta.

Os resultados de umidade do suco de caju em pó variaram de 1,36 a 3,35\% e estando de acordo com os parâmetros estabelecidos pela legislação para produtos desidratados, sendo este um fator de proteção ao desenvolvimento de microrganismos deteriorantes e patogênicos (Brasil, 2005).

Na Tabela 2 são apresentados os resultados da ANOVA a nível de $95 \%$ de confiança para as variáveis respostas de 
Tabela 1. Planejamento composto central rotacional e resultados médios do parâmetro de rendimento do pó do suco integral de caju

\begin{tabular}{|c|c|c|c|c|}
\hline \multirow[t]{2}{*}{ Ensaios } & \multicolumn{2}{|c|}{$\begin{array}{c}\text { Planejamento } \\
\text { composto central }\end{array}$} & \multicolumn{2}{|c|}{$\begin{array}{c}\text { Análises } \\
\text { realizadas (\%) }\end{array}$} \\
\hline & $X_{1}$ & $\overline{X_{2}}$ & Rendimento & Umidade \\
\hline A & $3,25(-1,00)$ & $130,00(-1,00)$ & 88,01 & 1,50 \\
\hline B & $3,25(-1,00)$ & $170,00(1,00)$ & 94,95 & 2,46 \\
\hline C & $4,25(1,00)$ & $130,00(-1,00)$ & 85,54 & 3,06 \\
\hline D & $4,25(1,00)$ & $170,00(1,00)$ & 98,31 & 2,04 \\
\hline$E$ & $3,04(-1,41)$ & $150,00(0,00)$ & 83,64 & 3,35 \\
\hline $\mathrm{F}$ & $4,46(1,41)$ & $150,00(0,00)$ & 83,61 & 2,71 \\
\hline G & $3,75(0,00)$ & $122,00(-1,41)$ & 73,98 & 3,05 \\
\hline $\mathrm{H}$ & $3,75(0,00)$ & $178,00(1,41)$ & 99,30 & 1,36 \\
\hline I & $3,75(0,00)$ & $150,00(0,00)$ & 93,20 & 3,00 \\
\hline $\mathrm{J}$ & $3,75(0,00)$ & $150,00(0,00)$ & 95,00 & 2,63 \\
\hline K & $3,75(0,00)$ & $150,00(0,00)$ & 94,40 & 2,74 \\
\hline
\end{tabular}

$\mathrm{X}_{1}$ - Vazão do ar quente $\left(\mathrm{L} \mathrm{min}^{-1}\right) ; \mathrm{X}_{2}$. temperatura de entrada do ar $\left({ }^{\circ} \mathrm{C}\right)$

Tabela 2. Análise de variância para os parâmetros de rendimento (\%) e umidade (\%) do pó do suco integral de caju

\begin{tabular}{lccccc}
\hline $\begin{array}{c}\text { Fonte de } \\
\text { variação }\end{array}$ & $\begin{array}{c}\text { Soma } \\
\text { quadrática }\end{array}$ & $\begin{array}{c}\text { Graus de } \\
\text { liberdade } \\
\text { Rendimento (\%) }\end{array}$ & $\begin{array}{c}\text { Média } \\
\text { quadrática }\end{array}$ & $\begin{array}{c}\text { Valor } \\
\text { de F }\end{array}$ & $\begin{array}{c}\text { Fcal/ } \\
\text { Ftab }\end{array}$ \\
Regressão & 474,43 & 5 & 94,89 & 3,89 & 0,77 \\
Residual & 122,12 & 5 & 24,42 & - & - \\
Falta de ajuste & 120,44 & 3 & 40,15 & 47,79 & 2,49 \\
Erro Puro & 1,68 & 2 & 0,84 & - & - \\
Total & 596,55 & 10 & - & - & - \\
\hline & \multicolumn{7}{c}{ Umidade (\%) } \\
Regressão & 2,69 & 5 & 0,54 & 1,77 & 0,35 \\
Residual & 1,52 & 5 & 0,30 & - & - \\
Falta de ajuste & 1,45 & 3 & 0,48 & 13,81 & 0,72 \\
Erro Puro & 0,07 & 2 & 0,04 & - & - \\
Total & 4,21 & 10 & - & - & - \\
\hline
\end{tabular}

$\mathrm{Ftab}=5,05$

rendimento e umidade do suco de caju em pó sinalizando que o modelo ajustado não foi significativo com coeficiente de correlação de 0,64 e 0,80, respectivamente. O teste F mostrou valores de Fcalculado inferior e superior aos valores de Ftabelado para a análise de regressão e falta de ajuste respectivamente para o parâmetro de rendimento e $\mathrm{F}$ calculado inferior aos valores de $\mathrm{F}$ tabelado tanto para a análise de regressão quanto para a falta de ajuste para o parâmetro de umidade.

Apesar do modelo não ter sido significativo verifica-se, observando os efeitos dos fatores lineares e quadráticos sobre o rendimento (Tabela 3) que apenas a vazão de ar quente em termo linear e a interação entre as variáveis independentes, não foram significativos sobre esta variável resposta, mas que somente a temperatura (linear) apresentou efeito positivo no rendimento, ou seja, um aumento qualquer neste parâmetro pode acarretar um aumento no rendimento do suco de caju em pó; já em termos quadráticos tanto a vazão de ar quente quanto a temperatura apresentaram efeito negativo sobre o rendimento.

Este efeito significativo da temperatura pode ser explicado pela maior eficiência no processo de transferência de calor e massa que ocorre quando maiores temperaturas de secagem são utilizadas. De acordo com Goula \& Adamopoulos (2005) temperaturas elevadas aumentam as taxas de secagem
Tabela 3. Efeito estimado, erro puro, coeficiente $t$, grau de significância estatística (p) para os fatores do modelo codificado para rendimento

\begin{tabular}{|c|c|c|c|c|}
\hline Fatores & $\begin{array}{c}\text { Efeito } \\
\text { estimado }\end{array}$ & $\begin{array}{l}\text { Erro } \\
\text { puro }\end{array}$ & $t(2)$ & p-valor \\
\hline & \multicolumn{4}{|c|}{ Rendimento (\%) } \\
\hline Vazão do ar quente (L) & 0,21 & 0,65 & 0,33 & 0,77 \\
\hline Vazão do ar quente $(Q)$ & $-7,23^{*}$ & $0,77^{*}$ & $-9,46^{*}$ & 0,01 * \\
\hline Temperatura (L) & $13,93^{*}$ & $0,65^{*}$ & 21,40 * & 0,00 * \\
\hline Temperatura (Q) & $-4,27^{\star}$ & $0,78^{*}$ & $-5,46^{*}$ & $0,03^{*}$ \\
\hline \multirow[t]{2}{*}{ Vazão X Temperatura } & 2,92 & 0,92 & 3,18 & 0,09 \\
\hline & \multicolumn{4}{|c|}{ Umidade (\%) } \\
\hline Vazão do ar quente(L) & 0,06 & 0,13 & 0,43 & 0,71 \\
\hline Vazão do ar quente $(Q)$ & 0,07 & 0,16 & 0,43 & 0,71 \\
\hline Temperatura (L) & $-0,61^{*}$ & $0,14^{*}$ & $-4,54^{*}$ & $0,04^{*}$ \\
\hline Temperatura (Q) & $-0,77^{\star}$ & $0,16^{*}$ & $-4,78^{*}$ & 0,04 * \\
\hline Vazão X Temperatura & $-0,99 *$ & 0,19 * & $-5,21$ * & 0,04 * \\
\hline
\end{tabular}

* Valores significativos a $p \leq 0,05$; $(\mathrm{L})$ : termo linear; $(\mathrm{Q})$ : termo quadrático

resultando em partículas mais secas ao colidirem com a parede interna da câmara de secagem do spray-dryer. Isto, por sua vez, acarreta em menor aderência do produto no interior do equipamento levando a maiores rendimentos de pó. Diante dos resultados apresentados nos ensaios da Tabela 1 foi constatado que o melhor resultado médio para o parâmetro de rendimento e o menor resultado médio para o parâmetro de umidade que correspondem ao ensaio $\mathrm{H}$, foram escolhidos como a melhor condição de processo.

Para a variável resposta de umidade observa-se, na Tabela 3 , que a temperatura (termos linear e quadrático) e a interação entre as variáveis independentes apresentaram efeito negativo sobre esta variável resposta, ou seja, um aumento qualquer neste parâmetro pode acarretar em diminuição na umidade deste pó. Efeito também observado por Tonon et al. (2008), em seu estudo sobre a secagem por atomização de suco de açaí. A Figura 1 mostra a superfície de resposta (A) e a curva de contorno (B) gerada através do modelo proposto para a variável resposta de rendimento evidenciando que os maiores valores encontrados para o parâmetro rendimento de processo ocorrem nas maiores temperaturas do ar. Apesar do delineamento não ter sido estatisticamente significativo, observa-se que foi encontrada uma região ótima experimental, ou seja, região na qual é obtido o maior percentual de rendimento não sendo possível se fazer mais testes com temperaturas maiores devido às características técnicas próprias limitantes do equipamento utilizado.

$\mathrm{Na}$ Tabela 4 se encontram os resultados das análises físicoquímicas no que concerne aos parâmetros de umidade, $\mathrm{pH}$, acidez, sólidos solúveis, vitamina $\mathrm{C}$ e parâmetros de cor tanto para o suco integral de caju como para o suco em pó obtido do ensaio $\mathrm{H}$ podendo-se dizer que o suco integral de caju se encontrava de acordo com os teores preconizados pelos padrões de identidade e na qualidade para este produto (Brasil, 2000).

Um dos fatores de proteção ao desenvolvimento de microrganismos deteriorantes e patogênicos é o teor de umidade enquanto o suco de caju em pó apresentou um valor médio de 1,04\%, constatando-se uma perda média de $98,84 \%$ de água quando comparada com a do suco integral de caju estando de acordo com o estabelecido pela legislação para 
A.

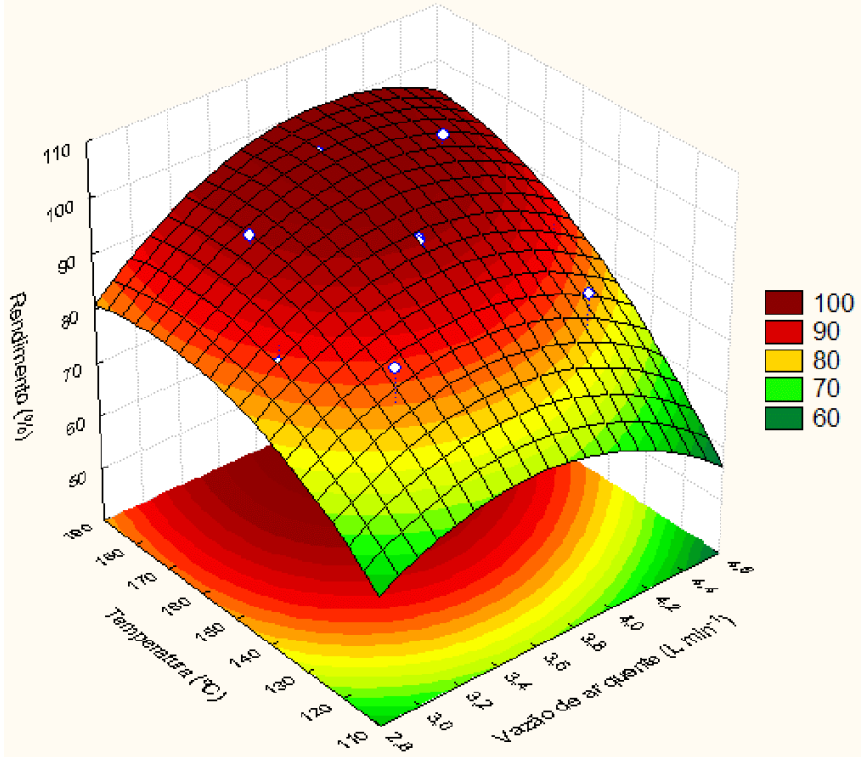

B.

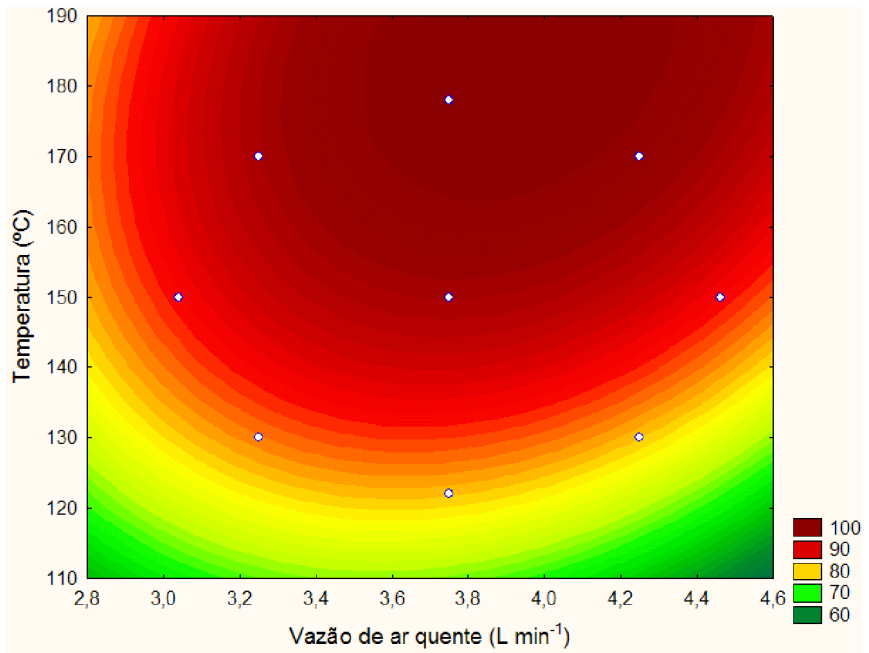

Figura 1. Superfície de resposta $(A)$ e curva de contorno (B) para a variável resposta de rendimento em função das variáveis independentes (vazão de ar quente e temperatura de entrada do ar) para o pó do suco de caju

produtos desidratados preconizando que produtos de frutas secos ou desidratados devem apresentar no máximo 25,00\% de umidade (Brasil, 2005). Este resultado foi inferior ao observado por Tonon et al. (2008), em seu estudo sobre a influência da temperatura do ar de secagem e da concentração de agente carreador sobre as propriedades físico-químicas do suco de açaí em pó, que encontraram um valor médio de 1,68\% com a utilização de $30 \%$ de maltodextrina e a temperatura do ar de secagem de $170{ }^{\circ} \mathrm{C}$.

Pinheiro et al. (2006) encontraram, em seu trabalho sobre sucos de frutas integrais, para o suco de caju valores de $\mathrm{pH}$ na faixa de 3,17 a 4,06 e os teores de sólidos solúveis variando no intervalo de 10,3 a 13 Brix, estando os resultados médios encontrados nesta pesquisa em concordância com os referidos autores. Com relação ao suco de caju em pó o pH apresentou valor inferior a 4,5 , outro fator que confirma a estabilidade deste
Tabela 4. Caracterização físico-química do suco integral de caju e do suco de caju em pó

\begin{tabular}{|c|c|c|c|c|}
\hline \multicolumn{2}{|c|}{ Parâmetros analisados } & Suco integr & & Suco em pó \\
\hline \multicolumn{2}{|c|}{ Umidade (\%) } & $90,02 \mathrm{a} \pm$ & 0,05 & $01,04 b \pm 0,01$ \\
\hline \multicolumn{2}{|l|}{$\mathrm{pH}$} & $3,92 \mathrm{a} \pm$ & 0,01 & $03,88 b \pm 0,01$ \\
\hline \multicolumn{2}{|c|}{ Acidez (\%) } & $0,31 \mathrm{a} \pm$ & 0,03 & $00,40 b \pm 0,01$ \\
\hline \multicolumn{2}{|c|}{ Sólidos Solúveis ( ${ }^{\circ}$ Brix) } & $10,60 \mathrm{a} \pm$ & 0,00 & $98,67 b \pm 0,58$ \\
\hline \multicolumn{2}{|c|}{ Vitamina C $\left(\mathrm{mg}^{\left.100 \mathrm{~g}^{-1}\right)}\right.$ b.s. } & $1.592,71 \mathrm{a} \pm 5$ & 52,05 & $282,67 b \pm 3,04$ \\
\hline \multirow{6}{*}{ Cor } & $L^{*}$ & $79,37 \mathrm{a} \pm$ & 0,03 & 0,01 \\
\hline & $a^{*}$ & $-3,64 \mathrm{a} \pm$ & 0,13 & $-1,52 b \pm 0,01$ \\
\hline & $b^{*}$ & $8,99 a \pm$ & 0,55 & $05,99 b \pm 0,01$ \\
\hline & $\mathrm{H}_{0}$ & $67,93 \mathrm{a} \pm$ & 0,89 & $75,73 b \pm 0,01$ \\
\hline & $C^{*}$ & $9,70 \mathrm{a} \pm$ & 0,54 & $06,18 b \pm 0,01$ \\
\hline & $\Delta \mathrm{E}^{*}$ & - & & $22,58 \pm 0,08$ \\
\hline
\end{tabular}

Médias seguidas da mesma letra na linha, não diferem entre si, segundo o teste $\mathrm{F}$ a $95 \%$ de confiança

produto pois produtos com tais valores de $\mathrm{pH}$ são considerados ácidos, dificultando o desenvolvimento e a proliferação dos microrganismos.

Observou-se também um incremento nos valores de acidez e sólidos solúveis ( ${ }^{\circ}$ Brix) no pó do suco de caju quando comparado com o suco de caju, fato que deve estar relacionado ao processo de secagem e, consequentemente, à concentração dos nutrientes no produto seco. Os valores de acidez, tanto para o suco integral quanto para o suco em pó, foram inferiores ao encontrado por Assis et al. (2007) em seu estudo de recuperação e concentração de componentes do aroma de caju (Anacardium occidentale L.) por evaporação.

Para o parâmetro vitamina C tanto para o suco integral de caju (1592,71 mg $100 \mathrm{~g}^{-1}$ em base seca ou 158,95 mg $100 \mathrm{~g}^{-1}$ em base úmida) quanto para seu pó, foram encontrados valores próximos aos da literatura por diversos pesquisadores, dentre os quais Cianci et al. (2005), Aragão \& Alsina (2007) e Maia et al. (2001). Ressalta-se que o processo de atomização ocasionou uma redução do teor de vitamina $\mathrm{C}$ da ordem de $82,25 \%$, redução relacionada ao efeito da temperatura na aceleração da degradação desta vitamina, fato documentado na literatura, por diversos autores, como por exemplo, Al-Zubaidy \& Khalil (2007), para suco de limão e Oliveira et al. (2013) em sua pesquisa sobre a cinética de degradação e vida de prateleira de suco integral de manga, além da presença de outros fatores que ocasionam esta perca como, por exemplo, a presença de oxigênio, luz etc.

O suco de caju em pó apresentou boa característica física de cor. Em relação a esta análise os parâmetros de luminosidade $L^{\star}$ foram de 79,37 e 57,09 para o suco integral e para o suco de caju em pó, respectivamente. Esta diferença de luminosidade pode estar relacionada ao processamento utilizado pois o efeito da temperatura durante o processo de secagem pode favorecer o escurecimento devido ao alto conteúdo de açúcar presente no suco de caju em pó. Este resultado está em concordância com os resultados observados por Quek et al. (2007), em seu estudo com suco de melancia em pó obtido em spray dryer.

Constatou-se, através dos parâmetros $\mathrm{a}^{\star} \mathrm{e} \mathrm{b}^{\star}$ que o suco de caju em pó apresentou tendência por croma $C^{\star}$ (parâmetro associado à pureza da cor) de menor intensidade de amarelo quando comparado com o teor observado para o suco de caju integral; já para o ângulo de tonalidade $\left(\mathrm{h}_{0}{ }^{*}\right)$ foi observado valor superior 
para o pó quando comparado ao do suco integral porém ambos os ângulos de tonalidade se situaram no primeiro quadrante do diagrama de cor, estando entre os eixos $a+$ (vermelho) $e+b$ (amarelo), tendendo mais ao amarelo que ao vermelho.

Segundo a National Bureau of Standard (NBS), a diferença de cor $(\Delta \mathrm{E})$ só é notada ao olho nu a partir de uma diferença de 0,5 entre a última medição e a primeira medição, ou seja, a amostra após a desidratação e a amostra in natura (Koksal \& Dikbas, 2008). Desta forma, a mudança de coloração para o pó do suco de caju pode ser detectada a olho nu, pois a diferença de cor ocorrida foi de 22,58, classificando esta mudança de coloração como grande alteração.

\section{Conclusões}

1. Não houve diferença significativa a nível de confiança de 95\%, no planejamento experimental utilizado revelando que o modelo não foi ajustado aos dados de acordo com a análise do teste $\mathrm{F}$ porém com a análise qualitativa foi possível estabelecer uma região ótima de processo.

2. O ensaio $\mathrm{H}$ foi escolhido como a melhor condição de processo seguindo os parâmetros de secagem: vazão de ar quente $\left(3,75 \mathrm{~m}^{3} \mathrm{~min}^{-1}\right)$, temperatura de entrada do ar (178 $\left.{ }^{\circ} \mathrm{C}\right)$, velocidade de alimentação $\left(0,55 \mathrm{~L} \mathrm{~h}^{-1}\right)$ e concentração de maltodextrina (30\%).

3. O suco integral de caju utilizado como matéria-prima para o desenvolvimento desta pesquisa se encontrava de acordo com os padrões preconizados pelos padrões de identidade e qualidade para este produto e a aplicação do processo de secagem em spray-dryer foi adequada para obtenção do suco de caju em pó com características físicas e físico-químicas de boa qualidade.

\section{Agradecimentos}

Os autores agradecem ao $\mathrm{CNPq}$, através do INCT, pelo recurso disponibilizado para a execução do projeto e à Universidade Federal do Ceará, pela utilização de sua infraestrutura.

\section{Literatura Citada}

Adhikari, B.; Howes, T.; Bhandari, B. R.; Troung, V. Effect of addition of maltodextrin on drying kinectics and stickiness of sugar and acid-rich foods during convective drying: experiments and modelling. Journal of Food Engineering, v.62, p.53-68, 2004.

Al-zubaidy, M. M. I.; Khalil, R. A. kinetic and prediction studies of ascorbic acid degradation in normal and concentrate local lemon juice during storage. Food Chemistry, v.101, p.254-259, 2007.

Andrigueto, J. R.; Nasser, L. C. B.: Teixeira, J. M. A. Sistema Agropecuário de produção integrada - SAPI. In: Conferência internacional sobre rastreabilidade de produtos agropecuários, 2, 2006, Brasília. Anais...Brasília: Ministério da Agricultura, Pecuária e Abastecimento, 2006. p.102-105,
Anselmo, G. C. S.; Cavalcanti-Mata, M. E. R. M.; Arruda, P. C.; Sousa, M. C. Determinação da higroscopicidade do cajá em pó por meio da secagem por atomização. Revista de Biologia e Ciências da Terra, v.6, p.58-65, 2006.

AOAC - Association of Official Analytical Chemists. Official methods of analysis of the AOAC. Washington: AOAC, v.2, 1997. 2p.

Aragão, R. F.; Alsina, O. L. S. Estudo experimental da secagem de fatias de caju em secador de bandejas. Alimentos Ciencia e Ingenieria, v.16, p.302-307, 2007.

Assis, A. V. R.; Bizzo, H. R.; Matta, V. M.; Cabral, L. M. C. Recuperação e concentração de componentes do aroma de caju (Anacardium occidentale L.) por pervaporação. Ciência e Tecnologia de Alimentos, v.27, p.349-354, 2007.

Brasil. Ministério da Agricultura, Pecuária e Abastecimento. Instrução Normativa no 1 , de 07 de janeiro de 2000. Dispõe sobre o "Regulamento técnico geral para fixação dos padrões de identidade e qualidade para polpa de fruta". Diário Oficial da União, Brasília: MAPA, 2000. p.54-58.

Brasil. Agência Nacional de Vigilância Sanitária. Resolução da Diretoria Colegiada - RDC n.272, de 22 de setembro de 2005. Dispõe sobre o "Regulamento técnico para produtos de vegetais, produtos de frutas e cogumelos comestíveis". Diário Oficial da União, Brasília: ANVS, 2005. 374p.

Cianci, F. C.; Silva, L. F. M.; Cabral, L. M. C.; Matta, V. M. Clarificação e concentração de suco de caju por processos com membranas. Ciência e Tecnologia de Alimentos, v.25, p.579-583, 2005.

Estrella, A. Sucos tropicais no Brasil para consumo local. In: Juice Latin America, 2004, São Paulo. Anais... São Paulo: [s.n.], 2004. CD-Rom

Goula, A. M.; Adamopoulos, K. G. Spray drying of tomato pulp in dehumidified air: I. The effect on product recovery. Journal of Food Engineering, v.66, p.25-34, 2005.

IAL - Instituto Adolfo Lutz. Normas analítica do Instituto Adolfo Lutz: Métodos químicos e físicos para análise de alimentos. 4.ed./1.ed. São Paulo: Instituto Adolfo Lutz, 2004. 1020p.

IBGE - Instituto Brasileiro de Geografia e Estatística. Produção agrícola municipal. 2012. <http://www.sidra.ibge.gov.br/ bda/tabela/listabl.asp? $\mathrm{z}=\mathrm{t} \& \mathrm{o}=11 \& \mathrm{i}=\mathrm{P} \& \mathrm{c}=1613>.5 \mathrm{Mar}$. 2014.

Koksal, T.; Dikbas, F. Color stability of different materials against various staining agents. Dent Materials Journal, v.27, p.139$144,2008$.

Maia, G. A.; Monteiro, J. C. S.; Guimarães, A. C. L. Estudo da estabilidade físico-química e química do suco de caju com alto teor de polpa. Ciência e Tecnologia de Alimentos, v.21, p.43-46, 2001.

Oliveira, A. N.; Ramos, A. M.; Chaves, J. B. P.; Valente, M. E. R. Cinética de degradação e vida-de-prateleira de suco integral de manga. Ciência Rural, v.43, p.172-177, 2013.

Pinheiro, A. M.; Fernandes, A. G.; Fai, A. E. C.; Prado, G. M.; Sousa, P. H. M.; Maia, G. A. Avaliação química, físicoquímica e microbiológica de sucos de frutas integrais: abacaxi, caju e maracujá. Ciência e Tecnologia de Alimentos, v.26, p.98-103, 2006. 
Quek, S. Y.; Chok, N. K.; Swedlund, P. The physicochemical properties of spray dried watermelon powder. Chemical Engineering and Processing. Lausanne, v.46, p.386-392, 2007. Statsoft. Statistica for Window - Computer programa manual. Versão 7.0 Tulsa: Statsoft Inc. 2007.
Tonon, R. V.; Brabet, C.; Hubinger, M. D. Influence of process conditions on the physicochemical properties of acai (Euterpe oleraceae Mart.) powder produced by spray drying. Journal of Food Engineering, v.88, p.411418, 2008. 